\title{
Drawing energy: evidence of next generation science standards for energy in diagrams
}

\author{
Kara E. Gray, and Rachel E. Scherr \\ Seattle Pacific University, Department of Physics, 3307 Third Avenue West, Seattle, WA, 98119
}

\begin{abstract}
The Next Generation Science Standards (NGSS) promote a model of energy that includes the ideas that energy is conserved, that energy can be tracked as a flow through a system, that energy transfer occurs through a variety of mechanisms, and other ideas. The NGSS also promotes the practice of developing and using representations of scientific concepts such as energy. We articulate the NGSS model of mechanical energy and translate it into a rubric for assessing energy diagrams. We assess the alignment of both professional and learner energy diagrams with the NGSS, and assess a class's increased facility with NGSS-aligned energy diagrams. The purpose of this research is to develop a tool for assessing students' ideas about energy and the effectiveness of energy instruction.
\end{abstract}

\section{INTRODUCTION}

The Next Generation Science Standards (NGSS) highlight energy as an essential concept in science [1]. This priority is demonstrated by energy's inclusion in both the cross-cutting concepts and the disciplinary core ideas. The NGSS promote a specific model of energy which emphasizes the conservation of energy, the tracking of energy through a system, and the mechanisms by which energy transfers and transforms. The NGSS also promotes the scientific practice of developing and using models or representations of scientific concepts. Representations such as diagrams help students visualize and understand abstract and complex scientific concepts and can provide a tool for assessment.

In this paper we articulate the NGSS model of mechanical energy and translate it into a rubric for assessing energy diagrams. We use this rubric to assess the alignment of four different energy diagrams with the NGSS: an energy bar chart, an energy flow diagram, an Energy Tracking Diagram [2], and a learner-invented diagram. Finally, we demonstrate how this rubric allows us to document a class's increased facility with NGSS-aligned energy representations. The purpose of this research is to develop a tool for assessing (1) students' use of the NGSS model in representations and (2) the effectiveness of energy instruction in supporting the NGSS model of mechanical energy.

\section{NGSS MODEL OF MECHANICAL ENERGY}

The NGSS model of mechanical energy is not expressed in a succinct statement; rather, it is expressed in a number of standards to be met at different grade levels. To discern the NGSS model of mechanical energy as a whole, we first made a complete list of every statement about mechanical energy in the NGSS and the Framework (from the Cross-Cutting Concepts and the following Disciplinary Core Ideas: 4-PS3A, 4-PS3-B, 4-PS3-C, MS-PS3-A, MS-PS3-B, HS-PS3-A, HS-PS3-B). Next, we identified the constituent ideas in each statement. For example, the standard "Energy can be moved from place to place by moving objects or through sound, light, or electrical currents" (4-PS3.A) includes concepts of (1) energy transfer ("energy can be moved from place to place”) and (2) mechanisms of energy transfer (“...through sound, light, or electrical currents”). We de-emphasized references to specific energy phenomena (such as sound, light, and electrical currents) in favor of statements contributing to the NGSS model of energy as a whole. We identified eleven basic ideas as constituting the NGSS model of mechanical energy. These eleven constituent ideas are the basis for evaluating the extent to which an energy diagram instantiates the NGSS model of energy. For each constituent idea, we identify in parentheses how that idea might be explicitly represented in a diagram.

1. Energy is conserved. (Energy units are pictured explicitly and the same number is visible throughout the scenario.)

2. Energy may be tracked as a flow among objects, fields, and systems. (Each energy unit comes from and goes to somewhere.)

3. Energy manifests in multiple forms. (Different types of energy are pictured.)

4. Forms of energy are indicated by observable quantities. (Observable quantities are associated with different forms of energy, e.g., warmth with thermal energy.)

5. Energy can transform from one form to another. (Energy units are shown changing from one form to another.)

6. Energy can transfer from one object, field, or system to another. (Energy units are shown moving from one object, field, or system to another.)

7. Energy transfer and transformation occurs through specific mechanisms or processes. (The means by which energy transfers or transforms is labeled, e.g., conduction, metabolism.) 
8. Any increase/decrease of energy in a system is due to energy entering/leaving the system. (A boundary is indicated that energy might cross, such that some energy is inside and some is possibly outside.)

9. Uncontrolled systems evolve toward more even energy distribution. (Energy spreads to more objects or energy goes to large objects, e.g., the air or the environment.)

10. Some forms of energy are less useful, e.g., thermal energy in the environment. (We do not assess energy usefulness [3] because we are not aware of a means by which this idea could be represented in a diagram.)

11. Mathematical expressions of conservation of energy may be used to predict and describe system behavior. (We do not assess energy quantification, which would show in mathematical expressions such as $1 / 2 \mathrm{mv}^{2}$.)

\section{ASSESSMENT OF SPECIFIC ENERGY DIAGRAMS}

In what follows, we illustrate the assessment of energy diagrams according to the rubric presented above. We assess an energy bar chart, an energy flow diagram, an Energy Tracking Diagram [2], and a learner-invented diagram. The first two diagrams are common textbook examples of energy representations. The third is a diagram developed to emphasize the flow of energy. The fourth diagram was chosen to illustrate the type of non-canonical diagrams learners can invent which instructors may need to assess. All but one of the diagrams depict the energy in the same "ring slider" scenario, in which a metal ring is smacked by a bentback ruler and slides across the floor (Fig. 1); these diagrams depict the energy of the ruler, ring, and environment (floor and air). (The energy flow diagram depicts a generalized engine scenario.)

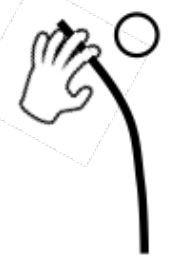

FIG. 1. Top view of ring slider scenario, in which a metal ring is smacked by a bent-back ruler and slides across the floor.

\section{A. Bar Chart}

A pair of bar charts for the ring slider scenario is shown in Fig. 2. The first chart depicts the energy when the ruler is bent back, before it smacks the ring; the second chart depicts the energy while the ring is sliding. This pair of bar charts depicts two features of the NGSS model of energy: Energy is conserved in that the height of the "total energy" bar is the same in both charts, and multiple forms of energy are depicted (kinetic, potential, and thermal).

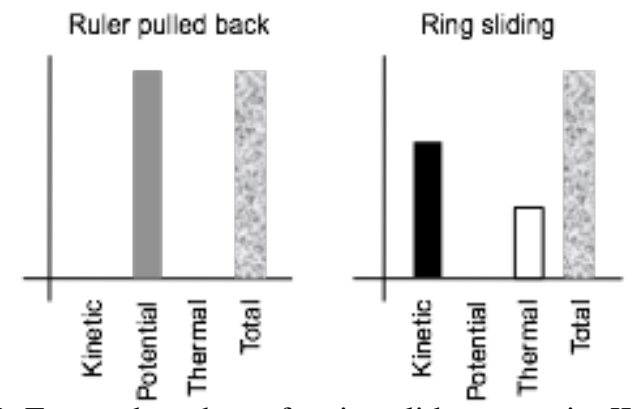

FIG. 2. Energy bar charts for ring slider scenario. While the ruler is pulled back (before it smacks the ring), all of the energy is potential energy. While the ring is sliding, the energy is partly kinetic (the ring is moving) and partly thermal (produced by the ring rubbing on the floor).

Several other features of the NGSS energy model, however, are not shown in this pair of diagrams. Energy is not $\mathbf{t}$ racked as a flow between objects, because no objects are depicted. Energy is not shown transferring from one object to another. Mechanisms of energy transfer are not indicated. Types of energy are not associated with observable quantities, i.e., thermal energy with temperature. Energy units are not shown as transforming from one form to another (only the distribution of energy among various forms is shown to change). Energy is not shown as moving toward a more uniform distribution among objects or in space, because no objects are depicted. Finally, a system is not depicted, in that there is no boundary indicated that energy might cross. (We might infer that the system is all the objects whose energy is included in the diagram - the ruler, the ring, and the environment-but this would be an inference; the system might equally well contain only the ruler and the ring, or only the ring.) Overall, bar charts are not well aligned with the NGSS model of energy.

\section{B. Energy Flow Diagram}

Energy flow diagrams are commonly used in the study of thermodynamics to represent the conversion of heat into work in heat engines and other devices. Figure 3 shows the energy flow for any continuously operating reversible device generating work from heat [4]. (The ring slider is not such a device.) This type of diagram depicts heat from a hightemperature reservoir $\left(Q_{h}\right)$ being input into a system (shown on the diagram as a gray box); some of this heat is converted into work $(W)$ that leaves the system, and some of the heat flows through the system to be output to a low-temperature reservoir $\left(Q_{l}\right)$. The quantity of energy flow is represented by the width of the arrows.

The diagram in Fig. 3 depicts many features of the NGSS model of energy. Energy is shown as being conserved in that the total width of the arrows is constant throughout the process. A system is explicitly shown, with energy crossing the boundaries of the system. Energy is tracked as a flow; though the objects among which it flows are not made 


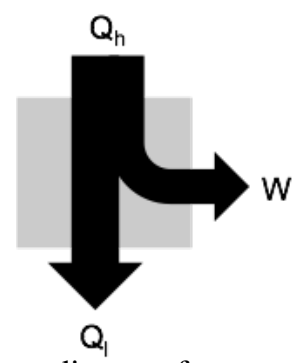

FIG. 3. Energy flow diagram for any continuously operating reversible device generating work from heat.

visible, the diagram shows transfer from one object to another (e.g., from the high-temperature reservoir to the lowtemperature reservoir) as well as mechanisms of transfer (e.g., heat and work). The diagram also shows the system evolving toward more uniform energy distribution, in that energy that started out in a single place is distributed to two places in the course of the scenario.

Other features of the NGSS model of energy are not depicted in Fig. 3. Forms of energy (such as thermal energy) are not shown; likewise, the diagram does not show transformations of energy (e.g., from thermal to kinetic), or associate energy forms with observable indicators such as temperature. Finally, this type of energy flow diagram focuses almost exclusively on the industrial conversion of heat to work, rather than supporting analysis of energy as a cross-cutting concept in physical, biological, chemical, and earth science scenarios [1]. Overall, though, energy flow diagrams of the type modeled in Fig. 3 align well with the NGSS model of energy.

\section{Energy Tracking Diagram}

In an Energy Tracking Diagram [2], objects are represented as schematic areas and individual energy units are represented as letters, with the specific letter representing the form of energy. Energy transfers and transformations are represented with arrows. The process or mechanism by which a transfer or transformation occurs (e.g., mechanical work) is represented by the color of the arrow. Relative amounts of energy may be represented by adding coefficients to the letters that represent units of energy.

Figure 4 is an Energy Tracking Diagram for the ring slider scenario. In this scenario, elastic energy in the ruler transforms into kinetic energy (the ruler moves), which transfers to the ring through mechanical work. That kinetic energy is then transformed into thermal energy in the ring and the floor as the ring slides across the floor. In the diagram, E, K, and T represent elastic, kinetic, and thermal energy; black, grey, and white arrows represent elastic expansion, mechanical work, and dissipation.

Energy Tracking Diagrams display many features of the NGSS model of energy. They show energy being conserved in that units of energy are pictured explicitly as persisting throughout the time development of the scenario. Energy is tracked as a flow among objects, with energy transfers

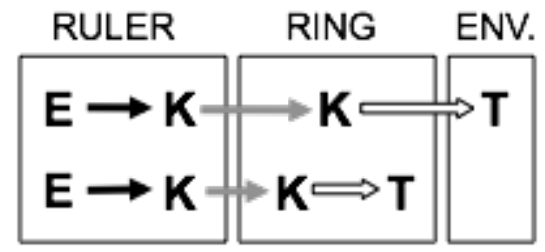

FIG. 4. Energy Tracking Diagram for ring slider scenario. $\mathrm{E}, \mathrm{K}$, and $\mathrm{T}$ represent elastic, kinetic, and thermal energy. Black, grey, and white arrows represent elastic expansion, mechanical work, and dissipation.

explicitly associated with specific mechanisms and processes. There are multiple forms of energy, and energy transformations are shown explicitly. Finally, energy is shown as moving toward a more uniform energy distribution, if we are willing to infer that the environment is physically large.

Energy Tracking Diagrams may be optionally modified to indicate a specific system by drawing a boundary around the objects to be included in the system. (For example, a boundary could enclose the ruler and ring together, or only the ruler.) Energy Tracking Diagrams do not typically include the observable quantities associated with specific forms of energy.

\section{Learner-Invented Diagram}

A learner-invented diagram of the energy in the ring slider is shown in Fig. 5. It shows elastic energy going to kinetic energy in the ruler, then going to kinetic energy in the ring. From there the energy goes into the floor and air as thermal, sound, and kinetic energy. The learner notes that deformation of the ring and floor are part of this process.

This diagram includes several NGSS features of energy. The diagram tracks energy as it starts in the ruler and then flows to other objects. Energy is transferred to multiple objects. Energy is represented using multiple forms of energy, and transforms from one form to another. The diagram shows the distribution of energy to several objects.

There are several NGSS features of energy not included in this diagram. The diagram does not show conservation of energy. Because units of energy are not shown, this diagram is not able to show that the amount of energy remains constant even as it is distributed to multiple objects. The diagram does not indicate a system, since no boundary is

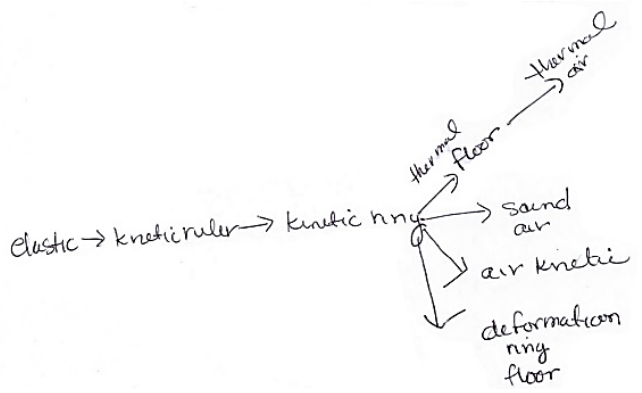

FIG. 5. Learner-invented diagram for the ring slider 
shown between objects inside and outside a boundary. The diagram does not show mechanisms of energy transfer or transformation: while transfers and transformations are indicated by arrows, they are not labeled. The diagram does include one mechanism, deformation, but doesn't tie it to a particular transformation. The diagram does not mention observables.

\section{ASSESSMENT OF A CLASS'S INCREASED FACILITY WITH ENERGY DIAGRAMS}

The rubric for assessing energy diagrams may be used to assess a class's increased facility with NGSS-aligned energy diagrams. In 2013, 15 secondary teachers participated in a second-year professional development (PD) course focused on the NGSS model of energy [5]. Learning goals included the development and use of NGSS-aligned energy representations such as Energy Tracking Diagrams [2,5]. Participants were given assessments before and after instruction, requesting energy analyses of the ring slider scenario (before), and a steam-turbine power plant (after). Before instruction only one diagram showed conservation of energy; at the end of the course, 12/15 diagrams did. Fewer than half of the learners' diagrams initially included transfers and transformation, whereas at the end of the course all diagrams included these features. Initially 9/15 diagrams included forms, while all diagrams did at the end. Initially 5/15 diagrams tracked energy; at the end, 14/15 diagrams did. Mechanisms of energy transfer and transformation were initially indicated in 5/15 diagrams; at the end of the course, 12/15 showed mechanisms. The number of diagrams showing distribution of energy went from $9 / 15$ to $14 / 15$. No diagrams explicitly indicated a system either before or after instruction. Explicit inclusion of observable indicators of energy decreased from 5/15 to $0 / 15$.

Application of the rubric shows that the class increased its facility with NGSS-aligned energy diagrams. This demonstrates that teachers can develop diagrams that are overall more aligned with the NGSS model of energy during PD. This analysis also demonstrates that this PD supported certain features of the NGSS model and deemphasized others. Fewer diagrams included indicators of energy at the end of the course than the beginning, possibly because learners did not consider this to be an important feature of energy diagrams.

\section{LIMITATIONS AND FUTURE RESEARCH}

There are important limitations to the rubric that we present for assessing learners' energy diagrams. First, this rubric does not assess a learner's energy model; rather, it assesses a learners' use of a specific representation of energy in a specific scenario. For example, a learner using a bar chart will not be able to track the flow of energy among objects no matter how rich their understanding may be, due to limitations imposed by the representation itself. Similarly, a learner analyzing a scenario involving only a single object will not be able to demonstrate understanding of energy transfers. Instructors should select representations and scenarios that foreground the features of the energy model that they want to assess. Another important limitation of the rubric is that it is almost certainly inappropriate to add scores on individual features to obtain an overall score (e.g., a to give a learner a score of $6 / 9$ if they demonstrate six of the nine features in the rubric). The nine items in the rubric are neither independent nor of equal weight. Subject to these limitations, the rubric supports assessment of learners' energy diagrams for alignment with the NGSS model of energy.

Future research will focus on two areas. First, we will analyze a wider variety of learner invented diagrams using this rubric in order to understand how learners' ideas about energy representation align with the NGSS model of energy. This is important since no canonical energy diagrams are closely aligned with the NGSS. Second we will analyze other PD courses to understand how the audience, instructional format, and learning goals affect learners' use of NGSSaligned energy diagrams.

\section{ACKNOWLEDGEMENTS}

We gratefully acknowledge Seattle Pacific University’s Physics Education Research Group. This material is based upon work supported by the National Science Foundation under Grants No. 0822342, 1222777, and 1418211.
[1] Next Generation Lead States (National Academies Press, Washington, DC, 2013); National Research Council (National Academies Press, Washington, DC, 2012)

[2] R. E. Scherr, H. G. Close, A. R. Daane, L. S. DeWater, B. W. Harrer, A. D. Robertson, L. Seeley and S. Vokos, Phys. Teach. 54 (2016)

[3] A. R. Daane, S. Vokos and R. E. Scherr, Phys. Rev. ST: Phys. Educ. Res. 10, 020111 (2014)
[4] For example, a similar diagram appears in C. Kittel and H. Kroemer (Freeman \& Co; New York), p. 229. (1980).

[5] R. E. Scherr, H. G. Close, S. B. McKagan and S. Vokos, Phys. Rev. - ST: Phys. Educ. Res. 8, 020114 (2012); R. E. Scherr, H. G. Close, E. W. Close and S. Vokos, Phys. Rev. - ST: Phys. Educ. Res. 8, 020115 (2012); A. R. Daane, L. Wells and R. E. Scherr, Phys.Teach. 52 (2014). 